\title{
Stoische Haltung, nach Epiktet
}

\section{Von Walter Siegfried}

Es ist unlängst das Wort von einer Restoisierung der Psychologie gefallen. So mag es wohl von einem gewissen Interesse sein, von neuem einen Blick auf die Lehre des Epiktet, eines Hauptvertreters der Stoa, zu werfen.

Epiktet lebte ungefähr von 60 bis 140; wurde in Hierapolis (Phrygien) geboren; war Sklave und wurde dann freigelassen; ließ sich in Rom nieder, wo er durch Musonius in die stoische Lehre eingeführt wurde; siedelte 94, als die Philosophen durch ein kaiserliches Dekret aus Rom verjagt wurden, nach Nikopolis (Epirus) über und war dort als vielbesuchter Seelsorger tätig. Seine Lehre wurde von seinem Schüler Arrian aufgezeichnet und unter dem Titel «Unterredungen» und in starker Verkürzung als «Handbüchlein» (der Moral) herausgegeben.

Auszugehen ist nach Epiktet von der fundamentalen Unterscheidung zwischen dem, was gänzlich und ausschließlich «bei uns steht», worüber letzten Endes einzig wir selber entscheiden und was uns daher unmittelbar «angeht», und dem, was nicht völlig oder überhaupt nicht «bei uns steht», was uns daher nicht unmittelbar, sondern - wenn überhaupt - bloß mittelbar «angeht».

Und was bei uns steht, ist seinem Wesen nach frei, keinem Zwang und keiner Behinderung unterworfen, es sei denn, da $\beta$ wir uns selber, zufolge einer schlechten Gewohnheit, einer verkehrten Auffassung usw., einen Zwang oder eine Behinderung auferlegt haben.

Wesensmäßig frei ist indessen nicht identisch mit faktisch frei, und es ist auch nicht im Sinne einer absoluten Ungebundenheit zu verstehen.

Als «vernunftbegabte», dem Rang nach gleich den Göttern «primäre», «um unsrer selbst willen» und nicht bloß als «Gebrauchsgegenstand» existierende Wesen sind wir andauernd, im Großen wie im Kleinen, vor die Alternative gestellt - und geben uns normalerweise auch «Rechenschaft» hievon -, uns richtig oder verkehrt zu verhalten, und mit der inneren Forderung konfrontiert, uns für das jeweils Richtige zu entscheiden, sei es für das, was bloß zweckmäßig und nützlich, sei es für das, was «naturgemäß», d. h. unbedingt richtig und somit rein an sich «schön» (absolut gut) ist und in jedem Fall «sich gebührt», kurz gesagt für das, was sein bzw. geschehen «sollte», und «schamhaft» alles zu vermeiden, dessen wir uns schämen müßten vor denen, die darum wissen. 
Diese Forderung können wir mißachten, aber wir können sie nicht aus der Welt schaffen.

Was richtig und was verkehrt ist, sagt uns die auch sich selber gegenüber kritisch eingestellte Vernunft, unser Leitorgan, ein abgespaltenes Teilchen von Gott, das dereinst zu ihm zurückkehren wird.

Sie ist auch der Aufseher, der uns untrüglich überwacht und dem nichts verborgen bleibt von dem, was wir denken und vorhaben. Somit ist in dem stoischen Begriff der Vernunft auch das miteinbeschlossen, was wir in unserem Bereich als Gewissen bezeichnen.

Von einer besonderen, von Gott auferlegten Strafe für schlechtes Verhalten ist bei Epiktet nirgends die Rede. Es genügt, daß wir mit solchem, den in uns wohnenden Gott besudelnden Verhalten uns selber bestrafen, indem wir damit unsere innere Haltung und Einstellung schädigen und uns unglücklich machen.

Und ebensowenig ist bei Epiktet von einer die menschliche Vernunft übersteigenden Offenbarung die Rede. Gott hat uns als Lehrmeister den Chrysipp und den Diogenes geschickt. Doch übersteigt ihre Botschaft keineswegs unsere Vernunft.

Im stoischen Sinne faktisch frei sind wir in dem Maße, als wir nicht zufolge einer verkehrten Auffassung, einer falschen inneren Bindung usw. uns selber daran hindern, das jeweils Rechte zu tun.

Zu dem, was nicht völlig bei uns steht und was uns daher nicht unmittelbar angeht und wodurch wir äußerlich in mannigfaltiger Weise gehemmt, gezwungen, gehindert sind, gehört zuerst einmal unser Körper, mit dem wir äußerlich «zusammengebunden» sind, der mancherlei Krankheiten ausgesetzt ist und unweigerlich einmal ausgedient haben wird;

sodann der Besitz, mit dem wir ebenfalls äußerlich «zusammengebunden» sind und den man uns wegnehmen kann;

ferner diejenigen Mitmenschen, mit denen wir auf die eine oder andere Weise äußerlich «zusammengebunden» sind, ihre Lebensdauer, wie sie uns einschätzen und sich dementsprechend verhalten zu uns;

ganz knapp ausgedrückt alles «Äußere», alles was nicht zu unserer inneren Haltung und Einstellung gehört.

Demgegenüber steht als unser Ureigenes völlig bei uns und kann von niemandem und durch nichts gehindert oder gezwungen werden und geht uns als einziges unmittelbar an die selbst gewählte und somit selbst gewollte innere Einstellung mitsamt der dazugehörigen inneren Haltung und seelischen Stimmung und dem ihr entsprechenden und entspringendem äußeren 
Tun - jene innere Einstellung und Haltung, die nicht einmal Zeus zu besiegen vermag.

$\mathrm{Da} \beta$ sie, für die wir im Alltag die Namen der verschiedenen Tugenden und ihres Gegenteils gebrauchen, unbedingt richtig ist und da $\beta$ wir sie unter allen äußeren Umständen im richtigen Zustand bewahren, muß unser erstes Anliegen sein. Denn wichtiger als was uns geschieht, ist die Art und Weise, wie wir uns einstellen und halten dazu.

Wir haben die Wahl, unsere Aufmerksamkeit in erster Linie auf unser Inneres zu richten und darob das Äußere in gewissem Sinne zu vernachlässigen oder dann uns ganz auf das Äußere zu konzentrieren und darob unser Inneres tatsächlich zu vernachlässigen.

Zur rechten inneren Einstellung gehört zuerst einmal, daß wir genau auf die Scheidung achten zwischen dem, was gänzlich bei uns steht und uns daher unmittelbar angeht, und dem, was nicht völlig oder gar nicht bei uns steht und uns daher nur mittelbar angeht, und uns streng davor hüten, etwas Fremdes fälschlicherweise für etwas Unsriges zu nehmen; denn damit wären wir immer wieder gehindert;

und daß wir uns von dem, was nicht völlig bei uns steht, (im richtigen Grad) «distanzieren», es (im richtigen Maße) «fahren lassen», es (im Vergleich zu dem, worauf es wesentlich ankommt) «für belanglos erachten», «uns nicht (in der Weise) darum kümmern» (als ob es die Hauptsache sei), statt es (in übertriebenem Maße) «wichtig zu nehmen» und «hochzuschätzen» und damit «in völliger Ergebenheit» uns (innerlich) total «festzubinden», «festzuknüpfen daran»; denn damit büßten wir unsere Freiheit ein;

daß wir ihm nur so viel an Aufmerksamkeit schenken, als ihm gewissermaßen als Material zukommt, wie der Bildhauer dem zur Verfügung stehenden Marmor, und es lediglich als Gelegenheit nehmen, unsere Kräfte zu üben, uns zu schmücken und zu zeigen, wer wir sind, und damit den andern ein Beispiel zu geben, was die rechte innere Haltung und Einstellung vermag;

und daß wir auf nichts Äußeres einen unbedingten Anspruch erheben, sondern uns zufriedengeben mit dem, was uns gewährt ist, und es dankbar als Geschenk auf Abruf entgegennehmen;

und $\mathrm{da} \beta$ wir uns innerlich als bloßen Teil einordnen in das große Ganze, unsern Willen in Einklang bringen mit dem, was uns widerfährt, indem wir es willig und sogar mit Wohlgefallen annehmen und ertragen, statt uns innerlich aufzulehnen dagegen und Götter und Menschen zu tadeln und ihnen Vorwürfe zu machen; 
und daß wir uns fest auf das Eigene konzentrieren, das, was bei uns steht, aufs Beste zu besorgen,

«bei der sich jeweils bietenden Materie das Primäre auszuarbeiten»;

«bei jeder Materie den jeweils gebührenden Ausweg, die gebührende Wendung zu suchen» und so an dem Platze, wo wir nun einmal hingestellt sind, als Bürger, Ehegatte, Sohn usw. die mit der äußeren Situation uns jeweils gestellte Aufgabe bestens zu erfüllen.

Dank dieser Einstellung sind wir frei; den «frei ist, wem alles nach seinem Willen (wohl besser; dem nichts entgegen seinem Willen) passiert, und den niemand zu hindern vermag»;

und imstande, jedes uns auferlegte Schicksal unbesiegbar, ohne uns dadurch entmutigen zu lassen, zu tragen, indem wir unberührt von Affekten die innere Ruhe und Überlegenheit bewahren und aufrecht und guten Mutes und heiter bleiben, was ausmacht, daß wir glücklich sind.

Zur rechten inneren Einstellung und Haltung gehört für Epiktet, gleichsam als Bestätigung aus höherer Warte, auch die Frömmigkeit, die rechte Einstellung zu Gott, unserem Vater.

Wir sollten uns dankbar und lobpreisend Rechenschaft geben vom Walten der göttlichen Vorsehung;

welche den Kosmos zu einem vollendeten Ganzen geordnet hat, in welchen die einzelnen Teile wunderbar aufeinander abgestimmt sind und welches bis ins Einzelne dauernd unter göttlicher Obhut steht und wo sogar das Böse seinen positiven Sinn hat, indem es uns Gelegenheit gibt, uns zu üben - was wäre aus Herakles geworden ohne den Nemeischen Löwen und ohne die Lernäische Hydra - und als von Gott berufene Zeugen den andern Menschen ein Beispiel zu geben, was die rechte innere Haltung und Einstellung vermag;

und welche uns die Fähigkeit gab, uns Rechenschaft zu geben von der göttlichen Ordnung,

und die Kraft, unser Schicksal würdig zu tragen, ohne uns dadurch entmutigen zu lassen,

und dafür sorgte, daß im extremen Ausnahmefall, wenn wir keine Möglichkeit mehr sehen, unser Leben einigermaßen «naturgemäß» zu verbringen, «uns die Tür offensteht», d.h. wir die Möglichkeit haben, freiwillig aus dem Leben zu scheiden.

Wir sollten Gott, und zwar einzig ihm, völlig ergeben sein, im Großen wie im Kleinen, einzig auf ihn schauen, uns so verhalten, wie es ihm wohlgefällig ist, 
ihm, was die Gesinnung betrifft, als unserem Vorbild nacheifern, uns unterordnend ihm gehorchen, unseren Willen in Einklang bringen mit ihm und mit dem, was er angeordnet hat,

indem wir willig und sogar mit Wohlgefallen annehmen und tragen, was er als Schicksal uns zugewiesen hat, seine Anweisungen streng befolgen und uns peinlich davor hüten, irgend etwas an der göttlichen Ordnung und Verwaltung zu tadeln statt Wohlgefallen zu finden daran.

Sich von seinem Körper, mit dem wir äußerlich «zusammengebunden», sind, innerlich zu distanzieren», bedeutet keineswegs, daß man ihn total vernachlässigen soll. Auch Sokrates und Diogenes vernachlässigten ihren Körper nicht. Wir brauchen ihn als notwendiges Werkzeug zum Leben und haben zu sorgen, daß er in gutem Zustande ist. Verkehrt ist lediglich, ihn in übertriebenem Maße «hochzuschätzen» und «wichtig zu nehmen» und damit uns innerlich total «festzubinden» an ihn, uns völlig «hinzugeben» an ihn und seine Gelüste. Denn damit geben wir andern Menschen eine Handhabe, uns zu knechten, und büßen so unsere Freiheit ein.

Desgleichen der Besitz, mit dem wir ebenfalls äußerlich «zusammengebunden» sind. Auch ihn brauchen wir als notwendiges Werkzeug zum Leben und sollten uns dementsprechend um ihn kümmern, jedoch ohne ihn übertrieben «wichtig zu nehmen» und ihm total «ergeben zu sein». Denn auch damit würden wir unsere Freiheit verlieren.

Seiner «Natur» nach ist der Mensch ein «geselliges» Wesen, was bedeutet, daß er auf Grund der ihm geschenkten Anlage gesellig sein sollte (Natur als Norm), im richtigen Fall tatsächlich so ist und sogar einen unwiderstehlichen Drang zur Geselligkeit hat.

Das bedeutet, daß richtigerweise wir menschenfreundlich sind, uns gegenseitig gern haben, einander zugeneigt sind und Freude haben am Umgang mit andern.

Und das bedeutet weiterhin, daß richtigerweise wir zuverlässig und vertrauenswürdig sind und bereit, unseren Mitmenschen, die kraft der gemeinsamen göttlichen Abstammung unsere Brüder sind, zu helfen, ihnen allerhand Wohltaten und Gefälligkeiten zu erweisen, ihre Unzulänglichkeiten geduldig zu ertragen und ihnen ihre Fehlgriffe zu verzeihen und, statt ihnen zu zürnen und womöglich Böses mit Bösem vergelten, sie lediglich bedauern und brüderlich, ohne Vorwurf und Tadel, zurechtweisen (correctio fraterna).

Auf Grund dieser Einstellung ist das Zusammenleben in Staat und Familie überhaupt erst möglich. 
Wie man es indessen mit jenen Elementen halten soll, die durch ihr kriminelles Verhalten das friedliche Zusammenleben zum mindesten erheblich stören, darüber gibt der erhaltene Text keine Auskunft.

Mit der Stellung, die wir innerhalb der menschlichen Gemeinschaft einnehmen, als Bürger, Familienvater usw., sind bestimmte Pflichten verbunden, die auf alle Fälle zu respektieren sind. Es darf z.B. nicht vorkommen, daß ein Vater, weil er glaubt, es bei seinem kranken Kind nicht mehr aushalten zu können, sich nach auswärts verzieht und erst auf die Nachricht hin zurückkehrt, es sei wieder gesund, und mit einem Vater hat man auf alle Fälle ehrerbietig umzugehen.

Daß der Mensch seiner Natur nach gesellig ist, bedeutet keineswegs, daß wir in totaler «Ergebenheit» uns innerlich an unsere Mitmenschen völlig «festbinden», uns ihnen total «angleichen» sollten. Vielmehr liegt es uns ob, ihnen ein Beispiel zu geben, was die rechte innere Haltung und Einstellung vermag.

Wie es verkehrt ist, uns innerlich total an sie festzubinden, so ist es umgekehrt ebenso falsch, sie gewissermaßen an uns festzubinden, indem wir tyrannisch verlangen, daß sie sich genauso verhalten, wie wir es wünschen, und «explodieren» und ihnen «zürnen», wenn dies nicht der Fall ist.

Auch sollte unsere Teilnahme am Geschick der Mitmenschen nicht so weit gehen, daß wir ihretwegen unglücklich sind. Denn keiner ist unglücklich durch einen andern. Und sollte es dennoch einmal passieren, so haben wir es einzig unserer verkehrten Einstellung zu verdanken.

Distanz wahren zu ihnen sollten wir auch in der Weise, daß wir uns in keinem Fall von ihnen abhalten lassen, das zu tun, was wir als unsere Pflicht erachten, wie wir auch ihnẹn die Freiheit gewähren sollten, sich nach ihrem eigenen Gutdünken zu verhalten, wobei indessen sich wiederum die Frage einstellt, ob diese Toleranz auch den kriminellen Elementen gegenüber zu wahren ist;

und da 3 wir niemandem in der Weise eine Handhabe bieten, uns zu knechten, daß wir begierig nach etwas streben, das sich nicht in unserer Gewalt befindet;

und daß wir ihnen, insbesondere den Mächtigen gegenüber, völlig unbefangen und furchtlos bleiben und uns zu keinerlei Schmeicheleien herbeilassen, in der Gewißheit, daß sie wohl über unseren Körper, unseren Besitz, jedoch niemals über unsere innere Einstellung und Haltung Gewalt haben, und daß, was sie an Ungutem uns antun, unmittelbar nur sie selbst und uns nur mittelbar angeht. 
Ferner steht völlig bei uns und sollte unbedingt richtig sein - denn davon hängt unsere innere Einstellung und Haltung ab - das wertende «Urteil», die wertende "Auffassung», m.a.W. die «Anwendung der Vorstellungen», welche darin besteht, daß wir die «zum voraus gefaßten», «uns angeborenen» «Vorstellungen» (wir sagen wohl besser «Begriffe») von gut und schlecht, schön und häßlich, usw. den jeweiligen konkreten Angelegenheiten «anfügen», wodurch diese uns als gut oder schlecht «erscheinen», wir sie uns als gut oder schlecht «vorstellen».

Kein Machtspruch kann mich zwingen, eine nach reiflicher Überlegung als wahr bzw. als falsch erkannte Aussage ehrlicherweise für falsch bzw. für wahr zu halten, ebensowenig wie jemand mich hindern kann, nach reiflicher Überlegung im Zweifelsfall mich ehrlicherweise eines Urteils zu enthalten.

Desgleichen kann z. B. niemand mich hindern, die von Bismarck publizierte Fassung der «Emser Depesche» ehrlicherweise für eine perfide Verfälschung zu halten. Wohl könnte jemand versuchen, mir dieses Urteil auszureden. Doch steht es letzten Endes bei mir, ob ich bei meiner Auffassung bleibe oder nicht.

Nach solchen Urteilen bestimmt sich unser Verhalten im Alltag.

«Ein jeglicher geht mit jeglichem so um, wie es der Auffassung entspricht, die er davon hat.»

«Bei einem jeden ist schuld, daß er etwas Bestimmtes tut (oder nicht tut), sein Urteil (daß es so richtig ist).»

«Weder der Tod, noch die Verbannung, noch die Mühsal, noch sonst etwas von der Art ist schuld daran, daß wir etwas Bestimmtes tun oder nicht tun, sondern unsere Auffassungen und Urteile ( $\mathrm{da} \beta$ der Tod usw. etwas Schlimmes sei).»

Falls jemand mich mit Androhung des Todes dazu zwingt, etwas Bestimmtes zu tun, so ist es (letztes Endes) nicht die Todesfurcht, die mich dazu zwingt, das Verlangte zu tun, sondern mein Urteil, es sei besser, das Verlangte zu tun als zu sterben.

«Was uns außer Fassung bringt oder hindert, sind unsere (verkehrten) Urteile, und nicht, was uns von außen geschieht.»

Für den Menschen ist maßgebend für alles, was er tut (oder nicht tut), daß es ihm als richtig (oder verkehrt) erscheint.

«Nichts anderes ist schuld an unserer Bestürzung und Verwirrung als unsere Urteile.»

«Wir selber sind es, die uns drücken und beugen, d.h. unsere Urteile drücken und beugen uns.» 
«Wenn wir etwas Verkehrtes tun, so wollen wir von nun an nichts anderes beschuldigen als unsere Urteile, auf Grund deren wir es getan haben.»

Ein jeder von uns verhält sich jeweils so, wie es unter einem selbstgewählten Aspekt - Pflicht, bloße Lust, materieller Vorteil usw. - ihn gut dünkt, ihm richtig scheint, er es sich als richtig vorstellt.

Wie es unmöglich ist, etwas Bestimmtes für falsch zu halten, was wir nach reiflicher Prüfung für wahr erkannt haben, ebenso können wir unmöglich etwas erstreben, das wir letzten Endes für schlecht halten.

Mit der Tötung ihrer eigenen Kinder tat Medea sich unsägliches Leid an. Doch fiel für sie letzten Endes stärker ins Gewicht, was sie damit dem treulosen Jason antat.

Auch die Geschichte im Großen wird durch solche Urteile, Auffassungen, Vorstellungen bestimmt. Hätte z.B. Paris es sich nicht als richtig vorgestellt, die Helena zu entführen, oder hätte Helena es nicht für richtig erachtet, ihm zu folgen, oder hätte etwas später Menelaos den Eindruck gehabt, es sei für ihn eigentlich ein Gewinn, solch eine Gattin loszuwerden, so hätte der zehn Jahre dauernde Trojanische Krieg nicht stattgefunden.

Beispiel aus unserer Zeit: Hätten nach der schmachvollen Entlassung des Generals v. Fritsch die Spitzen der Wehrmacht es für richtig erachtet, den sogenannten Führer samt dessen engeren Kumpanen zu liquidieren, so wäre vermutlich der Zweite Weltkrieg uns erspart geblieben.

Dasselbe gilt auch für die großen Gestalten der Dichtung.

Alles Unheil unter den Menschen kommt letzten Endes davon her, daß wir es nicht verstehen, die gemeinsamen, zum voraus gefaßten Begriffe von gut und schlecht usw. den konkreten Angelegenheiten in richtiger Weise anzufügen.

Aus der Verschiedenheit der Urteile können die größten Zwistigkeiten entstehen, wie das Beispiel von Achilleus und Agamemnon zeigt.

Daher ist es von der größten Wichtigkeit, die unser Verhalten regulierenden Urteile immer wieder zu prüfen, die schlechten auszumerzen und durch bessere zu ersetzen, und zwar so, daß man die daraus sich ergebenden Konsequenzen ins Auge faßt. Als verkehrt stellen sich unsere Urteile dann heraus,

wenn sie bei genauem Überlegen einen Widerspruch enthalten;

wenn wir durch sie etwas tun, was wir im Grunde genommen gar nicht wollen;

wenn wir durch sie geknechtet und unglücklich sind;

wenn durch sie das friedliche Zusammenleben gestört ist. 
Insbesondere gilt es, sich von den spontan «auf uns fallenden» Vorstellungen nicht zu einem Verhalten fortreißen zu lassen, das uns im Augenblick als richtig erscheinen mag, das wir jedoch gegebenenfalls nachträglich tief bedauern.

Absolut, bedingungslos gut ist nach Epiktet einzig die rechte innere Haltung und Einstellung samt dem ihr entsprechenden und entspringenden äußeren Tun, und bedingungslos schlecht das Gegenteil hievon, und alles Übrige dem absoluten Wert nach «belanglos», was indessen nicht ausschließt, ihm mit Rücksicht auf bestimmte Umstände einen relativen Wert zuzuschreiben.

Ein jedes Lebewesen ist letzten Endes am meisten sich selber zugetan, tut alles (letzten Endes) um seiner selbst willen, ist (letzten Endes) auf seinen eigenen Vorteil bedacht. Wir sollten in der Weise egoistisch sein, daß wir für uns das Beste, nämlich die rechte innere Haltung und Einstellung, wählen. Als Wesen, die dazu bestimmt sind, mit andern Menschen zusammenzuleben, verfehlen wir dieses Beste, wenn wir nicht bereit sind, unsern Beitrag für das Wohl der Gemeinschaft zu leisten.

Einzig mit dieser Auffassung sind wir in der Lage, die mit unserer sozialen Stellung verbundenen Pflichten zu erfüllen.

Und einzig mit dieser Auffassung wahren wir unsere Freiheit. Wer den Besitz oder eine bestimmte berufliche Stellung oder sonst etwas Äußeres für absolut gut und somit für etwas unbedingt zu Erstrebendes oder zu Bewahrendes hält oder wer seinem eigenen Leben einen unbedingten Wert zuschreibt, wird nicht umhin können, denen, die darüber verfügen, zu schmeicheln und ihnen zu Willen zu sein.

Von besonderer Wichtigkeit ist auch die Einschätzung unerer eigenen Person. Wer sich selbst ganz tief einschätzt, dem wird es z.B. nichts ausmachen, sich mit einem Betrug eine Menge Geld zu verschaffen, während ein anderer sich zu gut hält hiefür, sich nicht «hergibt» dazu.

Ferner steht bei uns und sollte unbedingt richtig sein und gehört ebenfalls zur inneren Einstellung das positiv gerichtete totale Streben, etwas Bestimmtes unbedingt zu erlangen, zu behaupten usw., sowie das negativ gerichtete totale Streben, etwas Bestimmtes unbedingt zu vermeiden, ihm unbedingt zu entgehen.

Wenn uns das so heiß Erstrebte entgeht, und gleichfalls, wenn wir in das hineingeraten, was wir unbedingt zu vermeiden trachten, sind wir unglücklich.

Und wer etwas heftig erstrebt, was nicht völlig bei ihm steht, wird 
abhängig von denen, die darüber verfügen, und somit genötigt, ihnen zu schmeicheln.

Daher sollten wir uns so einrichten, da $\beta$ wir mit dem positiven wie mit dem negativen totalen Streben in der Zone dessen verbleiben, was völlig und einzig bei uns steht, wo niemand und nichts uns hindern oder zwingen kann und wo der Erfolg oder Mißerfolg einzig von uns abhängt, mithin in der Zone dessen, was zu unserer inneren Haltung und Einstellung gehört.

An zwei Stellen empfiehlt Epiktet - offenbar im Hinblick auf die Anfänger in der philosophischen Unterweisung -, sich mit dem negativ gerichteten totalen Streben zu begnügen, d.h. heißt also, all das unbedingt zu vermeiden, was der rechten inneren Haltung und Einstellung widerspricht.

Als letztes steht völlig bei uns und sollte unbedingt fehlerlos sein der positiv gerichtete «Anstoß», den wir selber uns geben, gleich jetzt etwas Bestimmtes zu tun, sowie der negativ gerichtet «Anstoß, gleich jetzt etwas Bestimmtes nicht zu tun, es zu unterlassen. Auf solchen «Anstößen» beruht unser praktisches Verhalten im Alltag.

Dasselbe gilt auch für das Vorhaben, die Absicht usw.

$\mathrm{Da} \beta$ ein Befehl, positiven oder negativen Inhaltes, der an uns ergeht, tatsächlich wird, bedarf es eines entsprechenden «Anstoßes» unsrerseits, den wir an und für sich auch unterlassen könnten. Es steht mir z. B. völlig frei, ein Fahrverbot zu respektieren oder mich darüber hinwegzusetzen und damit eben eine Buße zu gewärtigen. Der Befehl bzw. das Verbot ist nichts weiteres als eine der vielen Situationen, mit denen wir äußerlich «zusammengebunden» sind und wo es darum geht, sich in aller Freiheit den richtigen Anstoß zu geben. Erst mit dem totalen, blinden, automatischen Gehorsam degradieren wir uns selbst zu einem vernunftlosen Gebrauchsgegenstand.

Sein Dasein auf stoische Weise zu verbringen ist keineswegs so einfach und leicht zu bewerkstelligen, wie es vielleicht den Anschein hat. Wie es nicht genügt, für ein Instrument begabt zu sein, um sogleich als ein großer Künstler dazustehen, die vorhandene Begabung vielmehr durch fleißiges Üben auszubilden und auf der jeweils erreichten Stufe zu unterhalten ist, und wie der Sportler dauernd trainieren muß, um in Form zu bleiben, ebenso muß die uns als Anlage geschenkte rechte innere Haltung und Einstellung erst noch erlernt, anerzogen, ausgebildet, eingeübt werden, wobei der angehende Stoiker sich zuerst einmal Rechenschaft geben muß von seinem bisherigen totalen Unvermögen in bezug auf das, was nottut, und dann mit Kleinem beginnen sollte. 
Zu dieser Selbsterziehung gehört, daß wir die Vorkommnisse des Alltags als Gelegenheit dazu benützen, um unsere rechte innere Haltung und Einstellung durch entsprechendes äußeres Verhalten zu bewahren und zu festigen,

und daß wir in strenger Selbstkontrolle verführerische Vorstellungen, die sich eingeschlichen haben, ausschalten, schlechte Gewohnheiten, z. B. den Jähzorn, durch entsprechende bewußt angenommene Gegengewohnheiten bekämpfen;

und daß wir regelmäßig, am besten frühmorgens, «meditieren», d.h. uns den einen und andern Fundamentalsatz, z. B. was uns unmittelbar angeht und was nicht, fest einprägen, ihn sogar schriftlich notieren, um ihn im Getriebe des Alltags, wo man so leicht seiner vergißt, stets bei der Hand zu haben,

und daß wir nach der Weise der Pythagoräer vor der Nachtruhe unser Verhalten während des vergangenen Tages einer strengen Prüfung unterziehen.

\section{Summary}

Epiktet was one of the main stoic philosophers (60-140 A. D.). He was born as a slave, but was set free later. He was teacher of philosophy in Rome and Nikopolis (Epirus). Of his works in the Greek language we possess the «Diatribai» («Entertainment») and the «Enchiridion» («Handbook» [of ethics]). The author of this article presents the most important principles of stoic comportment according to Epiktet.

Dr. Walter Siegfried

Spiegelhofstraße 35

CH-8032 Zürich 
